[CONTRIBUTION FROM THE CHEMICAL LABORATORY OF ClaRK UNIVERSitY, I, 15]

\title{
THE CONDUCTANCE OF SODIUM IODIDE IN AMYL ALCOHOL AT VERY LOW CONCENTRATIONS
}

\author{
By Charles A. Kraus and J. E,GBert Bishop 1 \\ Received July 14, 1922
}

\section{Introduction}

The present investigation grew out of an attempt to study the properties of dilute solutions of calcium chloride in amyl alcohol. In the course of this investigation great difficulty was experienced in obtaining solutions whose conductance did not vary as a function of the time, and it was accordingly decided to investigate the behavior of dilute solutions of sodium iodide as a preliminary to further investigation of calcium chloride solutions.

Solutions of sodium iodide in amyl alcohol have been investigated by Dutoit and Duperthuis ${ }^{2}$ and Keyes and Winninghoff ${ }^{3}$ at $25^{\circ}$. The results obtained in these investigations, however, do not agree well as regards the absolute value of the conductance of solutions in isoamyl alcohol, although in both instances it was found that the mass-action law appears to be approached as a limiting form at low concentrations. It was determined, therefore, to measure the conductance of sodium iodide in amyl alcohol at concentrations as low as $10^{-5} \mathrm{~N}$, if possible. It was hoped that results might be obtained which would serve as a test of the applicability of the mass-action law to concentrations much below those at which measurements have previously been carried out.

\section{Materials and Apparatus}

The isoamyl alcohol employed was prepared from a quantity of ordinary amyl alcohol. After an initial fractionation, the portion which came over at $130^{\circ}$ was dehydrated by means of quicklime, after which it was redistilled. The product was treated with a small amount of metallic sodium and the resulting solution repeatedly fractionated. By means of this treatment, it was possible to obtain amyl alcohol having a specific

1 The present investigation was carried out during the academic year 1916-17. In June, 1917, Mr. Bishop entered the Government Service and was stationed at the Picatinny Arsenal in charge of research throughout the period of the war. In the spring of 1919 , while carrying out experiments at the Arsenal, Mr. Bishop lost his life through an explosion resulting from a defective shell casing. Mr. Bishop had before him a very promising career in scientific work. In his untimely death science has lost one who possessed a vivid interest in its further development.

The present paper was prepared from Mr. Bishop's notes and manuscript which he left upon entering the Government Service. As may be seen, the investigation is incomplete in several directions. Nevertheless, the results obtained are of such a character that their publication appears well worth while. C. A. K.

${ }^{2}$ Dutoit and Duperthuis, J. chim. phys., 6, 699 (1906).

${ }^{3}$ Keyes and Winninghoff, Tris JOURNAL, 38, 1178 (1916). 
conductance of $2 \times 10^{-8}$; and by carrying out the fractionation under reduced pressure it was possible to obtain a product having a specific conductance as low as $1 \times 10^{-8}$. It was found, however, that solutions made up with this amyl alcohol were not stable, the conductance changing with the time. It appeared, furthermore, that the conductance change was the greater, the higher the specific conductance of the alcohol. It was determined, therefore, to attempt to purify the alcohol still further. For this purpose, the alcohol was subjected to fractional condensation under reduced pressure. The initial portion of the alcohol condensed was rejected and subsequent portions were introduced directly into the conductivity cell without coming in contact with ordinary air. By this means it was possible to obtain alcohol having a specific conductance as low as $1.4 \times 10^{-9}$. The lowest value previously recorded is $1.5 \times 10^{-8}$ by Keyes and Winninghoff. With alcohol of this low value of the specific conductance, it was possible to carry the measurements to concentrations as low as $10^{-5} \mathrm{~N}$, without an unduly large correction for the conductance of the pure solvent. It is interesting, too, to note that the solutions in alcohol prepared in this way were comparatively stable. For example, a solution of sodium iodide having a concentration of $17.3 \times 10^{-5}$ had an initial specific conductance of $13.744 \times 10^{-7}$. At the end of 14 hours the specific conductance of this solution was found to be $13.694 \times 10^{-7}$. In 14 hours, therefore, the specific conductance varied less than $0.4 \%$.

In the actual experiments, the specific conductance of the alcohol in the cell as used varied from $2.5 \times 10^{-8}$ to $5.4 \times 10^{-9}$. In all cases, during the course of a run, the solutions were found to be stable within the limits of the experimental error.

The sodium iodide employed was prepared from the best c. P. product obtainable upon the market and was purified by 4 recrystallizations from conductivity water. The crystallizations were carried out in the absence of oxygen in order to avoid oxidation.

The conductance was measured by means of a Kohlrausch bridge of the Leeds and Northrup pattern, a Vreeland oscillator being employed as source of alternating current and a telephone as an indicating instrument. A resistance box with Curtis-wound coils was employed, and the effects of inductance and capacity were balanced out with an air condenser.

All measurements were carried out at $25^{\circ}$ in a thermostat regulated to $0.005^{\circ}$. However, owing to defective operation of the temperature regulating mechanism, the temperature fluctuations amounted to as much as $0.02^{\circ}$ in the seventh run, and to $0.01^{\circ}$ in the eighth run.

The conductance cell consisted of a glass cylinder $25 \mathrm{~cm}$. long having a capacity of approximately $500 \mathrm{cc}$. At one end, a smaller tube having a diameter of approximately $2.5 \mathrm{~cm}$. with a length of $15 \mathrm{~cm}$. was sealed to the larger tube at right angles to its axis. Into this tube was sealed 
a second tube carrying the electrodes, somewhat after the manner of a Dewar tube. To the other end of the cell, a side arm was attached"through which was introduced the pure solvent directly from the still and later the electrolyte in the form of a more concentrated solution in amyl alcohol. After introducing the electrolyte, the contents of the cell were thoroughly mixed. This operation was readily carried out with the form of cell employed.

When alcohol having a specific conductance of $5 \times 10^{-9}$ or less was employed, it was found that the previous treatment of the cell had considerable influence on the specific conductance of the alcohol. Treating the cell with live steam appeared to leave behind a small amount of conducting material on the electrodes which resulted in a marked increase in the specific conductance of the solvent. This method of treatment was therefore discarded and after each run the cell was washed out a number of times with pure amyl alcohol and finally allowed to soak in the pure solvent for a time.

\section{Experimental Results}

The experimental results are given in the following table, in which the concentration of the electrolyte is given in the first column, the specific conductance in the second column, the equivalent conductance in the third column, and the values of the ionization function $K$, as calculated according to the equation $K=\frac{C \Lambda^{2}}{\Lambda_{0}\left(\Lambda_{0}-\Lambda\right)}$, in the fourth column. All readings taken are here given excepting 2 runs, which have been omitted because of known sources of error. The readings in the seventh series of experiments are subject to some error owing to temperature fluctuation as stated above. At the head of each series of measurements is given the value of $\nu$, the specific conductance of the pure solvent, which was subtracted from that of the solution in order to obtain the conductance due to the salt.

\section{TABLE I}

Conductance of Sodrum Iodide Solutrons in Iso-Amyl Alcohol at Different CONCENTRATIONS AT $25^{\circ}$

$\begin{array}{rrrr}C \times 10^{5} & \text { Spec. cond. } \times 10^{7} & \Lambda & K \times 10^{4} \\ & \text { Series } 1 & \nu=4 \times 10^{-9} & \\ 2.012 & 2.089 & 10.383 & 2.903 \\ 4.339 & 4.243 & 9.779 & 2.928 \\ 9.080 & 8.084 & 8.903 & 3.018 \\ 17.343 & 13.744 & 7.925 & 3.142 \\ & \text { Series 2 } & \nu=5.4 \times 10^{-9} & \\ 1.656 & 1.738 & 10.495 & 2.924 \\ 3.321 & 3.340 & 10.057 & 3.031 \\ 6.101 & 5.746 & 9.419 & 2.981 \\ 10.541 & 9.165 & 8.695 & 3.048\end{array}$




$\begin{array}{rrrr} & \text { Series 3 } & \nu=3.6 \times 10^{-9} & \\ 1.643 & 1.726 & 10.505 & 2.959 \\ 4.395 & 4.292 & 9.766 & 2.931 \\ 8.702 & 7.795 & 8.958 & 3.004 \\ & \text { Series } 4 & \nu=3 \times 10^{-9} & \\ 3.339 & 3.349 & 10.030 & 2.952 \\ 7.414 & 6.802 & 9.174 & 2.993 \\ 13.290 & 11.063 & 8.324 & 3.044 \\ & \text { Series } 5 & \nu=3.05 \times 10^{-9} & \\ 1.104 & 1.176 & 10.649 & 2.762 \\ 2.150 & 2.227 & 10.357 & 2.971 \\ & \text { Series } 6 & \nu=2.54 \times 10^{-9} & \\ 1.060 & 1.132 & 10.682 & 2.900 \\ 2.184 & 2.257 & 10.332 & 2.900 \\ 4.253 & 4.167 & 9.799 & 2.932 \\ 8.492 & 7.631 & 8.986 & 2.991 \\ & \text { Series } 7 & \nu=3.3 \times 10^{-9} & \\ 1.914 & 1.999 & 10.444 & 3.069 \\ 5.032 & 4.853 & 9.710 & 3.159 \\ 9.274 & 8.238 & 8.883 & 3.167 \\ 16.060 & 12.934 & 8.053 & 3.133 \\ & \text { Series } 8 & \nu=4.6 \times 10^{-9} & \\ 2.205 & 2.279 & 10.336 & 2.946 \\ 5.725 & 5.440 & 9.502 & 3.007 \\ 12.238 & 10.352 & 8.459 & 3.045\end{array}$

\section{Discussion of Results}

The results given in the preceding table are shown graphically in the accompanying figure, where values of the specific conductance are plotted as abscissas against those of the reciprocal of the equivalent conductance as ordinates. As is well known, if the law of mass action holds true, the experimentally determined points should lie on a straight line. As may be seen from the figure, in the more dilute solutions the points undoubtedly approach a linear relation. Even at the highest concentration, the deviations from a straight line are comparatively small. It is evident, however, that at the higher concentrations the points fall consistently somewhat below the straight line determined by the points in the more dilute solutions. Up to a concentration of $4.5 \times 10^{-5} \mathrm{~N}$, the deviation of the points from a straight line falls practically within the limits of the experimental error. The largest deviations are found in the first 2 points of Run 7, which is somewhat uncertain, as mentioned above, and the second point of Run 2. For the first 14 points, for which the concentration lies below $4.5 \times 10^{-5}$, the mean deviation of the conductance values from the straight line is $0.08 \%$. The deviation for 2 points lies between 0.2 and $0.3 \%$, for 4 points between 0.1 and $0.2 \%$ and for 8 points between 0.0 and $0.1 \%$. Considering the fact that the concentrations were carried as low as $1 \times 10^{-5} \mathrm{~N}$ and considering the difficulties encountered in the 
manipulation, the agreement would appear to be as good as might be expected. The curve extrapolated, assuming a linear relation, gives for $1 / \Lambda_{0}$ the value 0.09042 and for $\Lambda_{0}$, correspondingly, the value 11.059 . The value of $\Lambda_{0}$ may be fixed within a few tenths of $1 \%$.

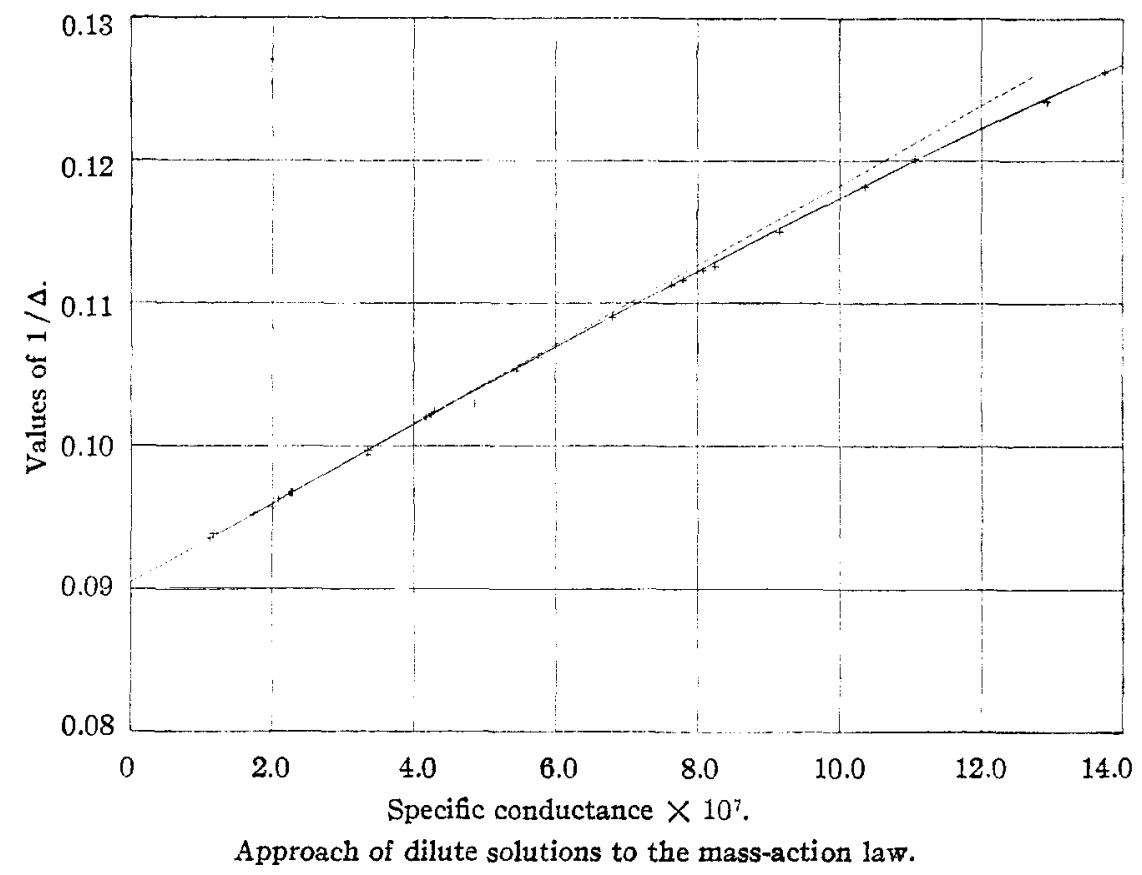

In the fourth column of the preceding table are given the values of the mass-action function as calculated with this value of $\Lambda_{0}$. As calculated from the plot, $K$ has a value $2.933 \times 10^{-4}$. The mean value of $K$ for the 14 points, up to a concentration of $4.5 \times 10^{-5} \mathrm{~N}$, is $2.936 \times 10^{-4}$. At higher concentrations, however, the deviations become appreciable, the function reaching a value of $3.14 \times 10^{-4}$ at a concentration of $17 \times 10^{-4}$.

While measurements in non-aqueous solutions have previously been carried to concentrations as low as $10^{-5} \mathrm{~N}$, the results have in no case been sufficiently precise to make it possible to determine with any considerable degree of certainty whether or not the results conform to the law of mass action at concentrations below $5 \times 10^{-5} \mathrm{~N}$. The results above given check even more closely than do the acids in water. It is noteworthy, too, that in the present instance the mean percentage accuracy is of the same order of magnitude over the entire concentration interval measured, thus indicating that the errors in dilute solutions, as in the more concentrated, are due to accidental causes and are for this reason not of a systematic nature. It must be concluded, therefore, that, in so far as conductance measurements have any significance in relation 
to the interpretation of the phenomena observed in dilute solutions of strong electrolytes in non-aqueous solvents, the mass-action law is approached as a limiting form at low concentrations, and that in solvents whose dielectric constants fall in the neighborhood of 15 the variation of the experimental values from the mass-action law amounts to only about $0.1 \%$ at concentrations below $4.5 \times 10^{-5} \mathrm{~N}$. In this connection, attention may be drawn to the fact that the more precise the experimental data, the more nearly do the results of conductance measurements conform to the requirements of the mass-action law at low concentrations. While it has not been possible, thus far, to confirm the results of conductance measurements in non-aqueous solutions by other methods, the uniform agreement of the results obtained by this method renders it highly probable that strong electrolytes in solution exist in a state of equilibrium and that at concentrations below $10^{-4} \mathrm{~N}$ this equilibrium conforms very nearly to requirements of the mass-action law. It is clear, too, that at higher concentrations the deviations become appreciable and are the greater, the greater the concentration. In all cases, the deviations are in the same direction, the value of the mass-action function increasing with increasing concentration. As the methods of measurement become more refined, the dilutions to which the experiments must be carried in order that the experimental values should conform to the mass-action law within the limits of experimental error become correspondingly greater. In the present instance, however, it has been shown that the points conform to the mass-action law within a very narrow limit of error over a considerable concentration interval.

On comparing the results of the present investigation with those of previous investigators, it is found that the value of $\Lambda_{0}$ is much higher than has been previously found for isoamyl alcohol. The values obtained by Keyes and Winninghoff, and by Dutoit and Duperthuis, according to Kraus and Bray, ${ }^{4}$ are 7.79 and 9.5 , respectively. The most probable explanation of this variation in the value of $\Lambda_{0}$ is on the assumption that there is present in the isoamyl alcohol prepared from technical products an isomeric alcohol which has a marked influence on the conductance. It is well known that ordinary fusel oil contains a considerable percentage of the opticallyactive amyl alcohol. According to the viscosity measurements of Thorpe and Rogers, it is not to be expected that the presence of even a considerable amount of the active isomer would have a large influence on the conductance of the resulting solution. At the present time, therefore, this question must be left open.

\section{Summary}

1. A method of preparing amyl alcohol of a high degree of purity is described, by means of which a solvent having a specific conductance

1 Krats and Bray, This JournaL, 35, 1315 (1913). 
of $1.4 \times 10^{-9}$ may be obtained. Solutions of sodium iodide in this alcohol appear to be entirely stable, varying only a fraction of $1 \%$ over a period of 14 hours.

2. The conductance of solutions of sodium iodide in amyl alcohol at $18^{\circ}$ was measured from a concentration of $1.0 \times 10^{-5}$ to approximately $17 \times 10^{-5} \mathrm{~N}$. The mean variation of the results does not exceed $0.1 \%$. In the more dilute solutions, the points conform to the requirements of the mass-action law within the limits of the experimental error. At the higher concentration there is a small although consistent deviation from the mass-action relation.

3. From an extrapolation of the plot to zero concentration there is obtained for $\Lambda_{0}$ the value 11.059 , and for $K$ the value $2.933 \times 10^{-4}$. The value of $\Lambda_{0}$ is not in agreement with that of Keyes and Winninghoff and of Dutoit and Duperthuis. The source of this discrepancy remains uncertain.

WORCESTER, MASSACHUSETTS

[CONTRIBUTION FROM THE CHEMISTRY DEPARTMENT OF THE UNIVERSITY OF WASHINGTON]

\section{THE ROLE OF HYDROGEN-ION CONCENTRATION IN THE PRECIPITATION OF COLLOIDS}

(Preliminary Report)

By Herman V. Tartar and Zalia Jencks Gailey

Received July 17, 1922

A large amount of experimental work has been done on the precipitation of colloids by electrolytes. An extensive review of the literature on this subject would be out of place here; for this information the reader is referred to standard texts on colloid chemistry and to certain papers, especially to Burton, ${ }^{1}$ Whitney and Ober, ${ }^{2}$ and Wo. Ostwald. ${ }^{3}$ In spite of the extensive work in this field, there are at present only a very limited number of generalizations and these are not quantitative. For this paper, it will suffice to point out and discuss briefly only those factors which have a very direct relation to the experimental results presented.

For many years it has been known that the precipitation of a suspensoid is determined primarily by the ion of the added electrolyte which has an electric charge opposite in sign to that of the colloidal particles. But different concentrations of salts containing the same precipitating ion are required for the precipitation of equal amounts of a given colloid; for example, different concentrations of potassium acetate and potassium

1 Burton, "The Physical Properties of Colloidal Solutions," Longmans, Green and Co., second ed., 1921, Chap. VIII.

${ }^{2}$ Whitney and Ober, THIs JournaL, 23, 842 (1901).

${ }^{3}$ Ostwald, $Z$. Kolloidchem., 26, 28 and 69 (1920). 